AEI-2009-085

\title{
Quasilocal equilibrium condition for black ring
}

\author{
Dumitru Astefanesei, Maria J. Rodriguez, and Stefan Theisen \\ Max-Planck-Institut für Gravitationsphysik, Albert-Einstein-Institut, 14476 \\ Golm, Germany \\ E-mail: dumitru@aei.mpg.de, maria.rodriguez@aei.mpg.de, \\ stefan.theisen@aei.mpg.de
}

\begin{abstract}
We use the conservation of the renormalized boundary stress-energy tensor to obtain the equilibrium condition for a general (thin or fat) black ring solution. We also investigate the role of the spatial stress in the thermodynamics of deformation within the quasilocal formalism of Brown and York and discuss the relation with other methods. In particular, we discuss the quantum statistical relation for the unbalanced black ring solution.
\end{abstract}

Keywords: Quasilocal formalism, Counterterms, Black Rings. 


\section{Contents}

1. Introduction 1

2. Quasilocal stress-energy tensor $\quad 3$

3. Unbalanced black ring $\quad 7$

$\begin{array}{lll}3.1 & \text { The model } & 7\end{array}$

3.2 Balance condition 8

4. Stresses and quantum statistical relation $\quad 10$

4.1 Gravitational tension 10

4.2 Generalized quantum statistical relation 12

5. Discussion 13

A. Stress tensor for boosted black string $\quad 14$ 


\section{Introduction}

A basic known fact of general relativity is that there is no concept of local energy for the gravitational field. Due to the equivalence principle it is possible to eliminate every observable effect of the gravitational field in a suitable spacetime neighborhood. ${ }^{1}$ Therefore, in general relativity the gravitational energy and momentum have non-local validity.

One of the most powerful frameworks for computing conserved quantities in general relativity is the 'quasilocal' formalism of Brown and York [1]. The basic idea in [1] is to define a 'quasilocal' energy inside a given finite region (rather than defining the energy at a point). Thus, an appealing feature of quasilocal energy is its direct derivation from the gravitational action for a spatially bounded region.

In this paper, we consider the quasilocal energy as applied to spacetimes that are asymptotically flat in spacelike directions. The spatial infinity — the part of infinity which is reached along spacelike geodesics - is represented by one point in the Penrose diagram of conformal compactification for Minkowski space. In this context, it is better to visualize it as the hyperboloid of spacelike directions (it is isometric to the unit 4-dimensional de Sitter space) — a detailed discussion on the role of boundary (conditions) for holography in flat spacetimes can be found in [2].

The relationship with the standard ADM [3] treatment at spatial infinity was presented in $[1,4]$. The quasilocal energy agrees with the ADM energy in the limit so that the spatial boundary is pushed to infinity.

It is also well known that the gravitational action contains divergences even at tree-level - they arise from integrating over the infinite volume of the spacetime. For any variational principle it is possible to add to the action terms that depend on the fixed boundary data. Thus, the action can be regularized by supplementing the quasilocal formalism by boundary terms (counterterms) (see [5] for asymptotically flat spacetimes ${ }^{2}$, [9] for asymptotically AdS spacetimes, [10] for a general dilaton potential and, e.g., [11] for AdS gravity with higher derivative terms) that depend on the intrinsic geometry of the regularizing surface. In this way the difficulties associated with the choice of a reference background are avoided.

Brown and York have also proposed a quasilocal stress-energy-momentum of gravitational field which is obtained by varying the action with respect to the metric on the boundary of the quasilocal region.

A concrete expression for the regularized 'boundary' stress-energy tensor when the spatial boundary is pushed to infinity was given in [12] — it was obtained from varying the action supplemented with the counterterms. This provides not just a concrete method to compute the conserved charges and to study thermodynamical

\footnotetext{
${ }^{1}$ Even if the gravitational field can be measured by the geodesic deviation of two observers, a single observer can not distinguish it from kinematical effects.

${ }^{2} \mathrm{~A}$ rigorous justification for using these counterterms can be found in $[6]$ (see also $[7,8]$ ).
} 
properties of black objects, but also gives the answer in a form which is holographic in spirit.

It is important to emphasize that, despite the similarity between the definitions of the boundary stress tensor $\left(\tau^{i j}\right)$ and the standard matter stress tensor $\left(T^{\mu \nu}\right)$, they have completely different physical interpretations [1].

First of all, it should be noticed that $\tau^{i j}$ characterizes the entire system, including contributions from both the gravitational field and the matter fields. On the other hand, for a background which satisfies the equations of motion for gravity and matter, the boundary stress tensor satisfies an approximate local conservation law [1]

$$
D_{i} \tau^{i j}=-T^{n j}
$$

Here, $D_{i}$ is the covariant derivative of the induced metric on the boundary and $T^{n j}=n_{\mu} T^{\mu j}$ ( $n_{\mu}$ is the normal on the boundary). The source term on the righthand side vanishes under the assumptions that matter fields fall off sufficiently fast at infinity (no matter in a neighborhood of the boundary). Obviously, when the component of $T^{n j}$ in the direction of a Killing vector $\xi^{i}$ vanishes, the matter fields do not play any role in defining the conserved charge associated with this Killing vector.

One would like to understand how this formalism is generalized to unbalanced solutions - this is especially interesting in connection with the black ring/blackfold approach $[13,14,15]$. In the black ring/blackfold approach, one can construct new black hole solutions in higher dimensions by using (thin) black branes curved into a specific shape. Thus, an important question is if the solution remains regular after bending.

We will see that for the unbalanced solutions there can also exist a source term in the right-hand side of (1.1) due to the conical singularities in the metric. Based on this observation, we will use the conservation of the boundary stress tensor to obtain the equilibrium condition for a general (thin or fat) black ring solution (the dynamic balance condition for a thin black ring in five dimensions was first observed in $[16,17]$ and obtained in higher dimensions in [13]).

In this paper, we also carry out some preliminary investigation of the thermodynamics of the unbalanced solutions and present a 'generalized' quantum statistical relation. In particular, we use the quantum statistical relation to read off the term due to the stresses. In the limit for which this term vanishes, we will obtain the dynamic balance condition, or, in other words, a 'balanced' ring solution with the usual quantum statistical relation. This can be regarded as an important check of our proposal that the equilibrium condition can be obtained from the conservation of the renormalized boundary stress tensor.

At this point it is worth to comment on the relation with the previous literature. The fact that the ring is not in equilibrium should be reflected in a non-conservation of the stress tensor was first pointed out in [13]. The thin black ring is approximated 
by a black string (a black 1-brane) ${ }^{3}$ and so, at large distances, the gravitational field is generated by a source with a distributional stress-energy tensor which has non-zero components only along directions tangent to the worldvolume. The main observation in [15] is that, for a thin brane, the equations of motion [18] can be obtained by demanding the conservation of the stress-energy tensor. Thus, the absence of the external forces in the equations of motion is equivalent with the conservation of the stress-energy tensor.

However, our situation is slightly different. First of all, we keep the conical singularity in the boundary and so the 'shape' of the boundary is changed - we compute the boundary stress tensor with respect to this metric. ${ }^{4}$ More importantly, we do not have to use the thin ring approximation, our computations are done for a general black ring solution. The non-conservation of the boundary stress tensor in our case should be related to the modification (the existence of conical singularities) of the standard asymptotics for flat spacetimes.

Consistent with the point raised above, it is important to emphasize that the stress tensor considered in $[13,15]$ is defined in the bulk. To make the connection with this work more concrete we note that, in principle, one could also consider the quasilocal boundary for a foliation with the ring topology hypersurfaces. If such an analysis is possible, it should capture the information about the stress of the conical disk for the unbalanced solution.

The structure of the paper is the following: in the next section we describe the quasilocal formalism and set the conventions for the rest of the paper. In Section 3 we obtain the balance condition by using the energy conservation of the boundary stress tensor. In Section 4 we propose a generalized quantum statistical relation for the unbalanced black ring and check consistency with the proposal in the previous section. Finally, we conclude with a discussion of our results. Some details about the boundary stress tensor of a boosted black string are collected in appendix.

\section{Quasilocal stress-energy tensor}

In this section we present a review of the quasilocal formalism, set the conventions for the rest of the paper, and comment on the role of energy conservation in understanding the unbalanced solutions.

In a very basic sense, gravitational entropy can be regarded as arising from the Gibbs-Duhem relation applied to the path-integral formulation of quantum gravity [20]. In the semiclassical limit this yields a relationship between gravitational entropy

\footnotetext{
${ }^{3} \mathrm{~A}$ ring can be obtained by bending a boosted black string [17].

${ }^{4}$ In ADM formalism, a computation of the energy for a background with a boundary which contains a conical singularity was presented in [19] - despite this similarity, our computations are not related in any way with the ones presented in this paper.
} 
and other relevant thermodynamic quantities, such as mass, angular momentum, and other conserved charges.

The conserved charges of asymptotically flat black holes are usually computed by using Hamiltonian methods. The gravitational Hamiltonian must have well defined functional derivatives and must preserve the boundary conditions on the fields. Since there is a formal connection between total energy and time translation, it is natural to expect that the gravitational mass should be related to the value of the gravitational Hamiltonian - this idea is at the basis of defining both, the ADM and quasilocal, masses.

The quasilocal energy is the value of Hamiltonian which generates unit magnitude proper-time translations in a timelike direction orthogonal to spacelike hypersurfaces at some fixed spatial boundary [1] — it agrees with the ADM energy in the limit that the spatial boundary is pushed to infinity.

However, it is important to point out a key difference between the quasilocal mass and the ADM mass [4]. In ADM formalism the hypersurfaces are Cauchy surfaces and so the data on one slice completely determines the future evolution of the system. That is not the case for the quasilocal formalism: the hypersurfaces with which we foliate the spacetime are not Cauchy surfaces.

In this paper, we are interested in 5-dimensional stationary solutions. We choose the observers that are stationary with respect to the boundary, i.e., their five-velocity is perpendicular to the normal. These are the observers that will not stretch or squash the boundary, which would affect the energy. Therefore, we define the asymptotically flat spacetimes to have the following fall off behavior [21]

$$
\begin{aligned}
d s^{2}=g_{\mu \nu} d x^{\mu} d x^{\nu} \simeq & \left(-1+\frac{8 M_{A D M}}{3 \pi r^{2}}+\mathcal{O}\left(r^{-3}\right)\right) d t^{2}-\left(\frac{8 J_{\phi} \sin ^{2} \theta}{\pi r^{2}}+\mathcal{O}\left(r^{-3}\right)\right) d t d \phi \\
& -\left(\frac{8 J_{\psi} \cos ^{2} \theta}{\pi r^{2}}+\mathcal{O}\left(r^{-3}\right)\right) d t d \psi \\
& +\left(1+\mathcal{O}\left(r^{-1}\right)\right)\left(d r^{2}+r^{2}\left(d \theta^{2}+\sin ^{2} \theta d \phi^{2}+\cos ^{2} \theta d \psi^{2}\right)\right)
\end{aligned}
$$

having the spherical spatial infinity, $S_{\infty}^{3}$. We use Greek indices to denote the bulk coordinates.

The validity of this metric in the asymptotic region could always be used to define mass and angular momenta, $M_{A D M}$ and $J_{a}$. We note that the quasilocal definitions are more powerful because they do not involve a particular coordinate system.

To define the conserved charges within quasilocal formalism, we use the divergencefree boundary stress tensor proposed in [12]:

$$
\tau_{i j} \equiv \frac{2}{\sqrt{-h}} \frac{\delta I}{\delta h^{i j}}=\frac{1}{8 \pi G}\left(K_{i j}-h_{i j} K-\Psi\left(\mathcal{R}_{i j}-\mathcal{R} h_{i j}\right)-h_{i j} \square \Psi+\Psi_{; i j}\right)
$$


where $\Psi=\frac{c}{\sqrt{\mathcal{R}}}, h_{i j}$ is the induced boundary metric, and $\mathcal{R}_{i j}$ its Ricci scalar. Note that the constant $c=\sqrt{2}, \sqrt{3 / 2}$ for a boundary topology $S^{2} \times R \times R$ or $S^{3} \times R$, respectively.

The renormalized action $I$ in five dimensions considered in the definition of this stress tensor, including the counterterms, is of the form

$$
I=\frac{1}{16 \pi G_{5}} \int_{M} R \sqrt{-g} d^{5} x+\frac{\epsilon}{8 \pi G_{5}} \int_{\partial M}(K-c \sqrt{\mathcal{R}}) \sqrt{-h} d^{4} x
$$

where $K$ is the extrinsic curvature of $\partial M, \epsilon=+1$ where $\partial M$ is timelike or $\epsilon=-1$ where it is spacelike, and $h$ is the determinant of the induced metric on $\partial M$. Also, the counterterm considered here involves the Ricci scalar $\mathcal{R}$ of the induced metric on the boundary $h_{i j}$.

As we have already mentioned in the Introduction, this stress tensor is covariantly conserved (with respect to the boundary metric) for vacuum regular solutions. When there are also matter fields, the equation (1.1) expresses the local conservation of the boundary stress-energy up to the flow of matter energy-momentum across the boundary.

The boundary metric can be written, at least locally, in ADM-like form

$$
h_{i j} d x^{i} d x^{j}=-N^{2} d t^{2}+\sigma_{a b}\left(d y^{a}+N^{a} d t\right)\left(d y^{b}+N^{b} d t\right)
$$

where $N$ and $N^{a}$ are the lapse function and the shift vector respectively and $\left\{y^{a}\right\}$ are the intrinsic coordinates on the hypersurface $\Sigma$. The boundary conditions for the quasilocal Hamiltonian include fixation of the boundary spatial metric, lapse function, and shift vector.

Provided the boundary geometry has an isometry generated by a Killing vector $\xi^{i}$, a conserved charge

$$
\mathfrak{Q}_{\xi}=\oint_{\Sigma} d^{3} y \sqrt{\sigma} n^{i} \tau_{i j} \xi^{j}
$$

can be associated with the hypersurface $\Sigma$ (with normal $n^{i}$ ). Physically this means that a collection of observers on the hypersurface whose metric is $h_{i j}$ all observe the same value of $\mathfrak{Q}_{\xi}$ provided this surface has an isometry generated by $\xi^{i}$.

The mass and the angular momenta are

$$
M=\oint_{\Sigma} d^{3} y \sqrt{\sigma} n^{i} \tau_{i j} \xi_{t}^{j}, \quad J_{\phi}=\oint_{\Sigma} d^{3} y \sqrt{\sigma} n^{i} \tau_{i j} \xi_{\phi}^{j}, \quad J_{\psi}=\oint_{\Sigma} d^{3} y \sqrt{\sigma} n^{i} \tau_{i j} \xi_{\psi}^{j}
$$

where the normalized Killing vectors associated with the mass and angular momenta are $\xi_{t}=\partial_{t}, \xi_{\phi}=\partial_{\phi}$, and $\xi_{\psi}=\partial_{\psi}$ respectively. As it was recently explicitly shown in several concrete 5-dimensional black objects examples [22], the conserved charges from the quasilocal formalism match the ADM charges exactly. 
Armed with this formalism we will obtain the balance condition for a general black ring solution in the next section. As an warm-up exercise let us discuss MyersPerry black hole [23] (with one angular momentum) in Boyer-Lindquist coordinates:

$$
\begin{aligned}
d s^{2}= & -d t^{2}+\Sigma\left(\frac{r^{2}}{\Delta} d r^{2}+d \theta^{2}\right)+\left(r^{2}+a^{2}\right) \sin ^{2} \theta d \psi^{2}+r^{2} \cos ^{2} \theta d \phi^{2} \\
& +\frac{m}{\Sigma}\left(d t-a \sin ^{2} \theta d \psi\right)^{2}
\end{aligned}
$$

where

$$
\Sigma=r^{2}+a^{2} \cos ^{2} \theta, \quad \Delta=\left(r^{2}+a^{2}\right) r^{2}-m r^{2}
$$

and $m$ is a parameter related to the physical mass of the black hole, while the parameter $a$ is associated with its angular momentum.

The metric above depends only on two of the coordinates: $0<r<\infty$ and $0<\theta<\pi / 2$ and is independent of time $-\infty<t<\infty$, and the angles $0<\phi, \psi<2 \pi$. Asymptotically the metric approaches the flat background

$$
d s^{2} \simeq-d t^{2}+d r^{2}+r^{2}\left(d \theta^{2}+\cos ^{2} \theta d \phi^{2}+\sin ^{2} \theta d \psi^{2}\right)
$$

The non-vanishing components of the stress tensor (2.2) when $c=\sqrt{3 / 2}$ are

$$
\begin{aligned}
\tau_{t t} & =\frac{1}{8 \pi G_{5}}\left(-\frac{3}{2} m \frac{1}{r^{3}}-\frac{5}{3} a^{2} \frac{\cos 2 \theta}{r^{3}}+\mathcal{O}\left(1 / r^{5}\right)\right) \\
\tau_{t \psi} \equiv \tau_{\psi t} & =\frac{1}{8 \pi G_{5}}\left(-2 a m \frac{\sin ^{2} \theta}{r^{3}}+\mathcal{O}\left(1 / r^{5}\right)\right) \\
\tau_{\theta \theta} & =\frac{1}{8 \pi G_{5}}\left(\frac{2}{3} a^{2} \frac{\cos 2 \theta}{r}+\mathcal{O}\left(1 / r^{3}\right)\right) \\
\tau_{\phi \phi} & =\frac{1}{8 \pi G_{5}}\left(\frac{2}{3} a^{2} \frac{(1+2 \cos 2 \theta) \cos ^{2} \theta}{r}+\mathcal{O}\left(1 / r^{3}\right)\right) \\
\tau_{\psi \psi} & =\frac{1}{8 \pi G_{5}}\left(\frac{2}{3} a^{2} \frac{(-1+2 \cos 2 \theta) \sin ^{2} \theta}{r}+\mathcal{O}\left(1 / r^{3}\right)\right)
\end{aligned}
$$

The stress-energy conservation law can be easily checked. For a generic boundary metric (2.8) (with $r$ constant) and a stress tensor with non-vanishing components as in (2.9), the only non-trivial term is

$$
D^{i} \tau_{i \theta}=\left[\partial_{\theta}\left(\sin \theta \cos \theta \tau_{\theta \theta}\right)+\tan ^{2} \theta \tau_{\phi \phi}-\cot ^{2} \theta \tau_{\psi \psi}\right](\cos \theta \sin \theta)^{-1}
$$

By replacing the explicit values from (2.9) one can easily check that $D^{i} \tau_{i \theta}$ vanishes.

This term is also playing an important role in our analysis of unbalanced solution. We will see in the next section that this term does not vanish when the boundary metric contains conical singularities - the 'non-conservation' of the boundary stress tensor measures the deviation from the standard boundary conditions. The presence of conical singularities in the boundary metric is not, however, a drastic change (it is not similar, e.g., with changing the asymptotics to Anti-de Sitter) and this is why we can still use the boundary stress tensor (2.2) to study this type of spacetime. 


\section{Unbalanced black ring}

In this section, we explicitly check the conservation of the regularized stress-energy tensor for the unbalanced black ring. We also show how the conservation law yields the dynamical equilibrium condition for any, thin (black ring solutions with parameters $\nu, \lambda<<1$ ) or fat (those that are not thin), singly spinning black ring. ${ }^{5}$

\subsection{The model}

Using the conventions in [16] we can write a general line element as

$$
\begin{aligned}
d s^{2}= & -\frac{F(x)}{F(y)}(d t+R \sqrt{\lambda \nu}(1+y) d \psi)^{2} \\
& +\frac{R^{2}}{(x-y)^{2}}\left[-F(x)\left(G(y) d \psi^{2}+\frac{F(y)}{G(y)} d y^{2}\right)+F(y)^{2}\left(\frac{d x^{2}}{G(x)}+\frac{G(x)}{F(x)} d \phi^{2}\right)\right]
\end{aligned}
$$

with

$$
F(\xi)=1-\lambda \xi, \quad G(\xi)=\left(1-\xi^{2}\right)(1-\nu \xi)
$$

$R, \lambda$ and $\nu$ are parameters whose appropriate combinations give the mass and angular momentum. The parameters $\nu$ and $\lambda$ have the range $0 \leq \nu<\lambda<1$. Asymptotic spatial infinity is reached as $x \rightarrow y \rightarrow-1$.

This is a vacuum solution of Einstein equations in five dimensions. To obtain a Lorentzian signature, the variables $x$ and $y$ are constraint to take values in

$$
-1 \leq x \leq 1, \quad-\infty<y \leq-1, \quad \lambda^{-1}<y<\infty
$$

Note that for $y \in\left[\lambda^{-1}, \nu^{-1}\right]$ the coordinate $t$ becomes spacelike and so $t$ and $y$ are interchanged.

It is also important to emphasize that, since there are conical singularities, this is not a regular solution. Once the conical singularities are eliminated, we get a physical regular solution (black hole or black ring).

As shown in [16], in order to balance forces in the ring one must identify $\psi$ and $\phi$ with equal periods

$$
\Delta \phi=\Delta \psi=\frac{4 \pi \sqrt{F(-1)}}{\left|G^{\prime}(-1)\right|}=\frac{2 \pi \sqrt{1+\lambda}}{1+\nu}
$$

This eliminates the conical singularities at the fixed-point sets $y=-1$ and $x=-1$ of the Killing vectors $\partial_{\psi}$ and $\partial_{\phi}$, respectively.

\footnotetext{
${ }^{5}$ Note that this classification is equivalent to the one employed in finding the higher dimensional thin black rings of [13] with $r_{0}<<R$, where $r_{0}$ is the radius of $S^{2}$ and $R$ is the radius of $S^{1}$. As shown in [16] a redefinition of the form $\nu=r_{0} \sinh ^{2} \sigma / R$ and $\lambda=r_{0} \cosh ^{2} \sigma / R$ gives the relationship between the parameters of the black string and the black ring while changing the coordinates $r=-R F(y) / y, \cos \theta=x, z=R \psi$ and taking the $R \rightarrow \infty$ limit.
} 
However there still is the possibility of conical singularities at $x=+1$. These can be avoided in either of two ways. Fixing

$$
\lambda=\lambda_{c} \equiv \frac{2 \nu}{1+\nu^{2}} \quad \text { (black ring) }
$$

makes the circular orbits of $\partial_{\phi}$ close off smoothly also at $x=+1$. Then $(x, \phi)$ parametrize a two-sphere, $\psi$ parametrizes a circle, and the solution describes a black ring. Alternatively, if we set

$$
\lambda=1 \quad \text { (black hole) }
$$

then the orbits of $\partial_{\phi}$ do not close at $x=+1$. Then $(x, \phi, \psi)$ parametrize an $S^{3}$ at constant $t, y$. The solution is the same as the spherical black hole of [23] with a single rotation parameter.

Both for black holes and black rings, $|y|=\infty$ is an ergosurface, $y=1 / \nu$ is the event horizon, and the inner, spacelike singularity is reached as $y \rightarrow \lambda^{-1}$ from above.

\subsection{Balance condition}

The equilibrium condition is a constraint on the parameters of the unbalanced ring solution [16] which is equivalent with the removing of all conical singularities in the metric.

A nice physical interpretation was given in [17]: the absence of conical singularities is equivalent with the equilibrium of the forces acting on the ring. A black ring can be obtained by bending a boosted black string. Thus, the linear velocity along the string becomes the angular velocity of the black ring. The equilibrium of centrifugal and gravitational forces imposes a constraint on the radius of the ring $R$, the mass, and the angular momentum. In this way one can see that, indeed, just two parameters are independent in the solution of the neutral black ring. A discussion of black string within the quasilocal formalism is presented in the following section.

The previous observation was used in $[16,17]$ and [13] to obtain a balanced condition for the thin rings. By using the conservation of the boundary stress tensor (2.2), we obtain the balance condition for a general (thin or fat) black ring solution.

The quasilocal formalism is employed in the asymptotic region and so only conical singularities in the boundary can be detected in the boundary stress tensor. Therefore, one should choose a general enough foliation so that the induced metric of the boundary contains this non-trivial information.

Unlike in the previous literature where the conical singularity in the boundary $x=y=-1$ was eliminated first, we will just get rid of the conical singularity in the bulk. Thus, to avoid the conical singularity in the bulk, one must identify $\psi$ and $\phi$ with an equal period

$$
\Delta \phi=\Delta \psi=\frac{4 \pi \sqrt{F(+1)}}{\left|G^{\prime}(+1)\right|}=2 \pi \frac{\sqrt{1-\lambda}}{1-\nu}
$$


For the reasons mentioned in Section 2 and since the computations simplify, we prefer to change the coordinates as follows:

$$
x \rightarrow-1+\frac{2 m \cos ^{2} \theta}{\left(r^{2}+a^{2} \cos ^{2} \theta\right)}, \quad y \rightarrow-1-\frac{2 m \sin ^{2} \theta}{\left(r^{2}-m+a^{2} \cos ^{2} \theta\right)}
$$

In order to get an asymptotic metric of the form (2.8), we work in the following gauge:

$$
m=\frac{(1+\lambda)^{2} R^{2}}{1+\nu}, \quad a=\frac{(1+\lambda)^{1 / 2} R(\lambda-1+\nu+3 \lambda \nu)^{\frac{1}{2}}}{(1+\nu)}
$$

To gain some intuition about these coordinates, let us consider a 5-dimensional spinning black hole with one angular momentum - it corresponds to $\lambda=1$ in (3.1). By the coordinate transformation (3.8) the solution takes exactly the form of (2.6) with the additional identification of parameters,

$$
m=\frac{4 R^{2}}{(1+\nu)}, \quad a=\frac{2^{3 / 2} R \sqrt{\nu}}{(1+\nu)}
$$

Now, let us consider the unbalanced solution (3.1) with the conical singularity in the bulk removed by (3.7). In the coordinates (3.8) the asymptotic form of the metric is

$$
\begin{aligned}
g_{t t} & =-1+\frac{2 R^{2} \lambda(1+\lambda)}{(1+\nu)} \frac{1}{r^{2}}+\mathcal{O}\left(1 / r^{4}\right) \\
g_{t \psi} & =\frac{2 R^{3}(1+\lambda)^{2} \sqrt{\lambda \nu(1-\lambda)}}{\left(1-\nu^{2}\right)} \frac{\sin ^{2} \theta}{r^{2}}+\mathcal{O}\left(1 / r^{3}\right) \\
g_{r r} & =1+\mathcal{O}\left(1 / r^{2}\right) \\
g_{\theta \theta} & =r^{2}+\mathcal{O}(1) \\
g_{r \theta} & =\mathcal{O}\left(1 / r^{3}\right) \\
g_{\phi \phi} & =\frac{(1-\lambda)(1+\nu)^{2}}{(1-\nu)^{2}(1+\lambda)} r^{2} \cos ^{2} \theta+\mathcal{O}(1) \\
g_{\psi \psi}= & \frac{(1-\lambda)(1+\nu)^{2}}{(1-\nu)^{2}(1+\lambda)} r^{2} \sin ^{2} \theta+\mathcal{O}(1)
\end{aligned}
$$

and the non-vanishing stress tensor components are

$$
\begin{aligned}
\tau_{t t} & =\frac{1}{8 \pi G_{5}}\left(-\frac{R^{2}(1+\lambda)(9 \lambda(1+\nu)-10(-1+\lambda-2 \nu) \cos 2 \theta)}{3 r^{3}(1+\nu)^{2}}+\mathcal{O}\left(1 / r^{4}\right)(\beta .1\right. \\
\tau_{\psi t} & =\frac{1}{8 \pi G_{5}}\left(-\frac{4 R^{3}(1+\lambda)^{5 / 2} \sqrt{\lambda \nu} \sin ^{2} \theta}{r^{3}}+\mathcal{O}\left(1 / r^{4}\right)\right) \\
\tau_{\theta \theta} & =\frac{1}{8 \pi G_{5}}\left(\frac{4 R^{2}(1+\lambda)(1-\lambda+2 \nu) \cos 2 \theta}{3 r(1+\nu)^{2}}+\mathcal{O}\left(1 / r^{3}\right)\right) \\
\tau_{\phi \phi} & =\frac{1}{8 \pi G_{5}}\left(\frac{4 R^{2}(1-\lambda)(1-\lambda+2 \nu) \cos ^{2} \theta(1+2 \cos 2 \theta)}{3 r(1-\nu)^{2}}+\mathcal{O}\left(1 / r^{3}\right)\right) \\
\tau_{\psi \psi} & =\frac{1}{8 \pi G_{5}}\left(\frac{4 R^{2}(1-\lambda)(1-\lambda+2 \nu)(-1+2 \cos 2 \theta) \sin ^{2} \theta}{3 r(1-\nu)^{2}}+\mathcal{O}\left(1 / r^{3}\right)\right)
\end{aligned}
$$


We find that, due to the existence of conical singularities, this stress tensor is not covariantly conserved with respect to the boundary metric (2.8) (with $r$ constant). In other words, the fact that the stress tensor is not conserved is reflected in the existence of additional stresses that deform (to some extent) the boundary metric.

We observe that the stress tensor is only conserved (is equivalent with the absence of external forces) when

$$
\lambda=\frac{2 \nu}{1+\nu^{2}}
$$

which is the dynamic equilibrium condition for black ring. We expect that this will also be confirmed for other unbalanced solutions such as the doubly spinning black ring [24] multi black hole solutions [25, 26, 27] and the new black holes of [28].

\section{Stresses and quantum statistical relation}

In this section we present a 'generalized' quantum statistical relation for the unbalanced black ring. We also explicitly obtain the dynamic equilibrium condition for the black ring in the limit for which the term due to the stresses vanishes.

For completeness, we start by presenting a brief review of the gravitational tension and discussing the black string solution (including the boundary conditions that permit variations of its length) (see e.g. [29] and references therein). In the second part of this section we compute the action of the unbalanced black ring and show that it satisfies a generalized quantum statistical relation.

\subsection{Gravitational tension}

We start by examining a boosted black string with a fixed length $L$ - the stress tensor components are presented in the Appendix. We obtain

$$
\lim _{r \rightarrow \infty} \sqrt{-h}(c \sqrt{\mathcal{R}}-K)=\frac{r_{0}}{2} \sin \theta+\mathcal{O}(1 / r)
$$

where $r_{0}$ is the event horizon and $\sigma$ the boost parameter in the black string solution (A.1), so the total action for the black string is

$$
I \equiv \beta G=\beta \frac{r_{0}}{4 G_{5}} L
$$

where $\beta=1 / T$ is the inverse of the temperature.

The other thermodynamical quantities, the linear mass density, the temperature, and the event horizon area per unit length are

$$
\frac{M}{L}=\frac{r_{0}}{4 G_{5}}\left(1+\cosh ^{2} \sigma\right), \quad T=\frac{(\cosh \sigma)^{-1}}{4 \pi r_{0}}, \quad \frac{\mathcal{A}}{L}=4 \pi r_{0}^{2} \cosh \sigma
$$


The quantum statistical relation contains an additional term

$$
G-(M-T S)=-\frac{r_{0} L \sinh ^{2} \sigma}{4 G_{5}}
$$

This non-trivial term can be interpreted as a new term, $v p$, where the linear momentum $p$ and the boost velocity $v$ are

$$
p=\frac{r_{0} L}{4 G_{5}} \cosh \sigma \sinh \sigma, \quad v=\tanh \sigma
$$

The boost velocity appears as an intensive parameter in the first law.

It is important to emphasize again that by bending a string to form a circle one can obtain a black ring with the horizon topology $S^{1} \times S^{2}$. One way to keep this configuration in equilibrium is to add angular momentum such that the repulsive centrifugal force balances the tension and gravitational self-attraction.

Since the boost velocity becomes the angular velocity after bending, one could intuitively guess that the equilibrium is obtained for some specific values of the boost parameter. Indeed, as observed in $[16,17]$ the equilibrium condition for a thin black ring is equivalent with demanding a vanishing tension for the black string in analogy with the ADM definition, within the quasilocal formalism the tension is proportional to the $\tau_{z z}$ component of boundary stress tensor. Using the value of $\tau_{z z}$ from the Appendix, we observe that only the tensionless black string with a boost parameter $\sinh ^{2} \sigma=1$ is a black ring in the thin limit.

The tension of a spacetime arises as an extension of the usual ADM gravitational charges when there are additional parameters that characterize the spacetime at infinity [30]. In this case, one can obtain two solutions with slightly shifted values of the parameters but with the same asymptotics [31, 32, 33].

A general definition for gravitational tension was given in [34] — however, as in the case of gravitational energy, it can only be defined with respect to a reference background. A definition of gravitational tension by using the renormalized boundary stress tensor is clearly more advantageous since the difficulties associated with the choice of a reference background are avoided.

Let us also briefly comment on the case of a static black string with the boundary conditions that permit variations of its length [31]. In this case, there is an additional 'work' term in the first law given by the product of the tension and the variation of the length at spatial infinity [30].

Since the computation of the boundary stress tensor for the static black string is similar with the one in Apendix, we do not present the results here. However, as expected, our results match the results in [31] and the quantum statistical relation contains a new term due to the tension.

More importantly, it was shown in [31] that, when the solutions are characterized by more than one modulus, the result can be simply stated in analogy with the physics 
of elastic materials [35]: the role of stresses is played by a tension tensor. Within the quasilocal formalism, the strain tensor should encode the changes in the boundary metric: in shape (e.g., when there exist conical singularities) and/or in size.

\subsection{Generalized quantum statistical relation}

We have shown in Section 3 that the conservation of the boundary stress tensor provides the required dynamical balance condition for the solution (3.1). However, the quasilocal formalism also provides a definition for the action which is related to the thermodynamical potential $G$. Thus, it is important to understand how the standard thermodynamics is changed for the unbalanced black ring solution.

To compute the renormalized action (2.3) we observe that the scalar curvature $R$ vanishes and so the only contributions are due to the surface terms. We obtain the following grand-canonical potential:

$$
G \equiv \frac{I}{\beta}=\beta \frac{k^{2} R^{2}}{16 \pi G_{5}} \frac{\lambda(1-\lambda)(1+\nu)}{(1-\nu)^{2}}
$$

We also compute the mass and the angular momentum

$$
M=\frac{3 k^{2} R^{2}}{16 G_{5} \pi} \frac{\lambda(1+\lambda)}{(1+\nu)}, \quad J_{\psi}=\frac{k^{2} R^{3}}{8 G_{5} \pi} \frac{(1+\lambda)^{5 / 2} \sqrt{\lambda \nu}}{(1+\nu)^{2}}
$$

where $k$ is the periodicity of $\phi$ and $\psi$, namely $\Delta \phi=\Delta \psi=k$. The event horizon, the temperature, and the angular velocity are

$$
\mathcal{A}=\frac{2 k^{2} R^{3}(1-\lambda) \sqrt{\lambda}(\lambda-\nu)^{3 / 2}}{(1-\nu)^{2}(1-\nu)}, \quad T=\frac{1}{4 \pi R} \frac{1-\nu}{\sqrt{\lambda(\lambda-\nu)}}, \quad \Omega=\frac{1}{R} \frac{(1-\nu)}{\lambda(1+\nu)} \sqrt{\left.\frac{\lambda \nu_{4}}{1-\lambda} 7\right)}
$$

Note that since we removed the bulk conical singularity and kept the one in the boundary metric, the charges differ from the ones in the original paper [16]. Of course, as the balance condition is imposed both results for the charges agree.

The balanced black ring solutions satisfy the standard quantum statistical relation

$$
G=M-T S-\Omega J_{\psi}
$$

In our case, the solution is not balanced and we obtain the following generalized quantum statistical relation:

$$
G-\left(M-T S-\Omega J_{\psi}\right)=\frac{k^{2} R^{2}}{16 G_{5} \pi} f[\nu, \lambda]
$$

It seems that the extra term, $f[\nu, \lambda]$, should correspond to the stresses due to the conical singularity in the boundary. This term can be written as

$$
f[\nu, \lambda]=\left(\frac{(1-\lambda)(3 \lambda+(\lambda-2) \nu)}{(1-\nu)^{2}}+\frac{2(1+\lambda)^{5 / 2}(1-\nu) \nu}{\sqrt{1-\lambda}(1+\nu)^{3}}-\frac{3 \lambda(1+\lambda)}{1+\nu}\right)
$$


It is zero for $\lambda=2 \nu /\left(1+\nu^{2}\right)$ which corresponds to the equilibrium condition for the asymptotically flat black ring. ${ }^{6}$

\section{Discussion}

In this work, we used the conservation of the renormalized boundary stress-energy tensor [12] to obtain the equilibrium condition for a general (thin or fat) black ring solution. This closes a gap left unanswered in the previous literature that dealt just with the thin ring.

We have also investigated the role of stresses in the thermodynamics of the unbalanced solutions and proposed a generalized quantum statistical relation for the unbalanced black ring.

The role of the spatial stress was already pointed out by Brown and York [1] where a discussion on the thermodynamics of static black holes with respect to a boundary at finite $r=R=$ constant (in the bulk) was presented. They provided an interpretation for the trace of spatial stress as a surface pressure. A straightforward generalization of this definition to (general) unbalanced solutions when the boundary is at infinity seems unlikely and we leave a detailed analysis of this non-trivial issue for future work.

The main goal of this work was to understand how the proposal of [13], that the dynamical balance of a thin black ring is related to the conservation of the stress tensor, can be extended to a general black ring solution. However, to reach this goal, we have used a slightly different method, namely the quasilocal formalism supplemented with the counterterms. The advantage of this method is that the problems associated with the background subtraction are avoided. Also, one can compute the action (on the Euclidean section) not just the conserved charges and so this method provides a complete way to study the thermodynamics.

A concrete example that was extensively studied in the literature was a black string with boundary conditions that permit variations of its length. The first law in this case can be expressed as

$$
d M=T d S+\Gamma d L
$$

where $\Gamma$ is the tension (it is proportional to $\tau_{z z}$ component of the spatial stress) and $L$ is the length of the string. ${ }^{7}$

\footnotetext{
${ }^{6}$ Note that all the other roots of $f[\nu, \lambda]=0$ that satisfy the condition $0 \leq \nu<\lambda<1$ do not correspond to (standard) asymptotically flat metrics.

${ }^{7}$ Since the mass is proportional to $L$, at first sight, the new term in the first law seems problematic. However, one can see that for holding a finite horizon area the mass parameter (that is the monopole in a multipoles expansion of $g_{t t}$ component of the metric) must be varied in a precise way as $L$ is varied. Thus, to get the correct first law one should express first the mass in terms of the horizon area and the length $L$ as independent variables [31].
} 
It seems that the only component of the spatial stress which plays a role in thermodynamics is $\tau_{z z}$, where $z$ is the direction along the string. Intuitively, one can easily understand that this component of the stress tensor is related to the tension of the string. However, it is not so obvious why the other components of the spatial stress do not play any role. In fact, one expects that when the boundary is at a finite distance in the bulk the analysis of Brown and York should also apply to black string and so all the components of the spatial stress should play a role. However, when the boundary is pushed to infinity the only relevant contribution is coming from the $\tau_{z z}$ component.

A connection between black strings and (thin) black rings was pointed out in $[16,17]$. That is the equilibrium condition for the (thin) black ring is equivalent to a vanishing tension of the black string. In other words, the only black strings that are obtained as a limit from black rings are the tensionless ones. This fact was further explored in $[13,15]$ and it was found that this connection is more subtle: the equilibrium condition can be obtained from the conservation of the stress-energy tensor. We have explicitly checked that the equilibrium condition for a general black ring solution can be obtained from the conservation of the renormalized boundary stress tensor.

By providing a generalized quantum statistical relation for the unbalanced black ring we also made a step in understanding the thermodynamics of unbalanced solutions. We have reached a similar conclusion as in [31] for extended objects. In our case the strain tensor encodes the modifications in the shape and size of the boundary metric. It is important to note, though, that our proposal can be used not just for extended objects (e.g., black strings), but also for more general (unbalanced) solutions.

As a final comment, we note that the quasilocal formalism supplemented with counterterms is a very robust method to study the thermodynamics of black objects and it may be useful in understanding the holography in flat space.

\section{Acknowledgments}

We would like to thank Oscar Varela for useful conversations. DA would also like to thank Niels Obers for interesting discussions. This work was supported by the German-Israeli Project cooperation (DIP H.52) and the German-Israeli Fund (GIF).

\section{A. Stress tensor for boosted black string}

A simpler perhaps but more intuitive example to understand the boundary stress tensor is the boosted black string 


$$
d s^{2}=-\hat{f}\left(d t-\frac{r_{0} \sinh \sigma \cosh \sigma}{r \hat{f}} d z\right)^{2}+\frac{f}{\hat{f}} d z^{2}+\frac{d r^{2}}{f}+r^{2}\left(d \theta^{2}+\sin ^{2} \theta d \phi^{2}\right)
$$

where

$$
f=1-\frac{r_{0}}{r}, \quad \hat{f}=1-\frac{r_{0} \cosh ^{2} \sigma}{r}
$$

The non-trivial components of the boundary stress tensor for a black string with a boost (parametrized by $\sigma$ ) in five dimensions are

$$
\begin{aligned}
\tau_{t t} & =\frac{1}{8 \pi G_{5}}\left(-\frac{r_{0}}{2}\left(1+\cosh ^{2} \sigma\right) \frac{1}{r^{2}}+\mathcal{O}\left(1 / r^{3}\right)\right), \\
\tau_{t z} & =\frac{1}{8 \pi G_{5}}\left(-\frac{r_{0}}{2} \cosh \sigma \sinh \sigma \frac{1}{r^{2}}+\mathcal{O}\left(1 / r^{3}\right)\right), \\
\tau_{\theta \theta} & =\frac{1}{8 \pi G_{5}}\left(-\frac{5}{8} \frac{r_{0}^{2}}{r}+\mathcal{O}\left(1 / r^{2}\right)\right), \\
\tau_{\phi \phi} & =\frac{1}{8 \pi G_{5}}\left(-\frac{5}{8} \frac{r_{0}^{2}}{r} \sin ^{2} \theta+\mathcal{O}\left(1 / r^{2}\right)\right), \\
\tau_{z z} & =\frac{1}{8 \pi G_{5}}\left(\frac{r_{0}}{2}\left(1-\sinh ^{2} \sigma\right) \frac{1}{r^{2}}+\mathcal{O}\left(1 / r^{3}\right)\right)
\end{aligned}
$$

The boundary stress tensor also satisfies the conservation law $D^{i} \tau_{i j}=0$. In this

case the covariant derivative is with respect to the following asymptotic black string metric (instead of (2.8) for black ring)

$$
d s^{2} \simeq-d t^{2}+d r^{2}+r^{2}\left(d \theta^{2}+\sin ^{2} \theta d \phi^{2}\right)+d z^{2}
$$

and the only non-trivial covariant derivative is

$$
D^{i} \tau_{i \theta}=\left[\sin \theta \partial_{\theta}\left(\sin \theta \tau_{\theta \theta}\right)-\cot \theta \tau_{\phi \phi}\right](\sin \theta)^{-2}
$$

Replacing (A.3) in the latter equation it is possible to check that the boundary stress tensor is indeed covariantly conserved for any value of the parameters in the solution.

\section{References}

[1] J. D. Brown and J. W. York, "Quasilocal energy and conserved charges derived from the gravitational action," Phys. Rev. D 47, 1407 (1993) [arXiv:gr-qc/9209012].

[2] D. Marolf, "Asymptotic flatness, little string theory, and holography," JHEP 0703, 122 (2007) [arXiv:hep-th/0612012].

[3] R. L. Arnowitt, S. Deser and C. W. Misner, "The dynamics of general relativity," arXiv:gr-qc/0405109. 
[4] J. D. Brown, S. R. Lau and J. W. York, "Action and Energy of the Gravitational Field," arXiv:gr-qc/0010024.

[5] S. R. Lau, "Lightcone reference for total gravitational energy," Phys. Rev. D 60, 104034 (1999) [arXiv:gr-qc/9903038];

R. B. Mann, "Misner string entropy," Phys. Rev. D 60, 104047 (1999) [arXiv:hep-th/9903229];

P. Kraus, F. Larsen and R. Siebelink, "The gravitational action in asymptotically AdS and flat spacetimes," Nucl. Phys. B 563, 259 (1999) [arXiv:hep-th/9906127].

[6] R. B. Mann and D. Marolf, "Holographic renormalization of asymptotically flat spacetimes," Class. Quant. Grav. 23, 2927 (2006) [arXiv:hep-th/0511096].

[7] R. B. Mann, D. Marolf and A. Virmani, "Covariant counterterms and conserved charges in asymptotically flat spacetimes," Class. Quant. Grav. 23, 6357 (2006) [arXiv:gr-qc/0607041].

[8] D. Astefanesei, R. B. Mann and C. Stelea, "Note on counterterms in asymptotically flat spacetimes," Phys. Rev. D 75, 024007 (2007) [arXiv:hep-th/0608037].

[9] M. Henningson and K. Skenderis, "The holographic Weyl anomaly," JHEP 9807, 023 (1998) [arXiv:hep-th/9806087];

V. Balasubramanian and P. Kraus, "A stress tensor for anti-de Sitter gravity," Commun. Math. Phys. 208, 413 (1999) [arXiv:hep-th/9902121];

K. Skenderis, "Asymptotically anti-de Sitter spacetimes and their stress energy tensor," Int. J. Mod. Phys. A 16, 740 (2001) [arXiv:hep-th/0010138].

[10] R. G. A. Cai and N. Ohta, "Surface counterterms and boundary stress-energy tensors for asymptotically non-anti-de Sitter spaces," Phys. Rev. D 62, 024006 (2000) [arXiv:hep-th/9912013].

[11] D. Astefanesei, N. Banerjee and S. Dutta, "(Un)attractor black holes in higher derivative AdS gravity," JHEP 0811, 070 (2008) [arXiv:0806.1334 [hep-th]].

[12] D. Astefanesei and E. Radu, "Quasilocal formalism and black ring thermodynamics," Phys. Rev. D 73, 044014 (2006) [arXiv:hep-th/0509144].

[13] R. Emparan, T. Harmark, V. Niarchos, N. A. Obers and M. J. Rodriguez, "The Phase Structure of Higher-Dimensional Black Rings and Black Holes," JHEP 0710, 110 (2007) [arXiv:0708.2181 [hep-th]].

[14] M. M. Caldarelli, R. Emparan and M. J. Rodriguez, "Black Rings in (Anti)-deSitter space," JHEP 0811, 011 (2008) [arXiv:0806.1954 [hep-th]].

[15] R. Emparan, T. Harmark, V. Niarchos and N. A. Obers, "Blackfolds," Phys. Rev. Lett. 102, 191301 (2009) [arXiv:0902.0427 [hep-th]].

[16] H. Elvang and R. Emparan, "Black rings, supertubes, and a stringy resolution of black hole non-uniqueness," JHEP 0311, 035 (2003) [arXiv:hep-th/0310008]. 
[17] R. Emparan, "Rotating circular strings, and infinite non-uniqueness of black rings," JHEP 0403, 064 (2004) [arXiv:hep-th/0402149].

[18] B. Carter, "Essentials of classical brane dynamics," Int. J. Theor. Phys. 40, 2099 (2001) [arXiv:gr-qc/0012036].

[19] U. Nucamendi and D. Sudarsky, "Quasi-asymptotically flat space-times and their ADM mass," Class. Quant. Grav. 14, 1309 (1997) [arXiv:gr-qc/9611043].

[20] G. W. Gibbons and S. W. Hawking, "Action Integrals And Partition Functions In Quantum Gravity," Phys. Rev. D 15, 2752 (1977).

[21] S. Tomizawa, Y. Yasui and A. Ishibashi, "A uniqueness theorem for charged rotating black holes in five-dimensional minimal supergravity," arXiv:0901.4724 [hep-th].

[22] D. Astefanesei, R. B. Mann, M. J. Rodriguez and C. Stelea, "Quasilocal formalism and thermodynamics of asymptotically flat black objects," arXiv:0909.3852 [hep-th]; D. Astefanesei, M. J. Rodriguez and S. Theisen, "Thermodynamic instability of doubly spinning black objects," arXiv:1003.2421 [hep-th].

[23] R. C. Myers and M. J. Perry, "Black Holes In Higher Dimensional Space-Times," Annals Phys. 172, 304 (1986).

[24] Y. Morisawa, S. Tomizawa and Y. Yasui, "Boundary Value Problem for Black Rings," Phys. Rev. D 77, 064019 (2008) [arXiv:0710.4600 [hep-th]].

[25] H. Elvang and P. Figueras, "Black Saturn," JHEP 0705, 050 (2007) [arXiv:hep-th/0701035].

[26] H. Iguchi and T. Mishima, "Black di-ring and infinite nonuniqueness," Phys. Rev. D 75, 064018 (2007) [Erratum-ibid. D 78, 069903 (2008)] [arXiv:hep-th/0701043]; J. Evslin and C. Krishnan, "The Black Di-Ring: An Inverse Scattering Construction," Class. Quant. Grav. 26, 125018 (2009) [arXiv:0706.1231 [hep-th]].

[27] H. Elvang and M. J. Rodriguez, "Bicycling Black Rings," JHEP 0804, 045 (2008) [arXiv:0712.2425 [hep-th]].

[28] H. Lu, J. Mei and C. N. Pope, "New Black Holes in Five Dimensions," Nucl. Phys. B 806, 436 (2009) [arXiv:0804.1152 [hep-th]].

[29] N. A. Obers, "Black Holes in Higher-Dimensional Gravity," Lect. Notes Phys. 769, 211 (2009) [arXiv:0802.0519 [hep-th]].

[30] J. H. Traschen and D. Fox, "Tension perturbations of black brane spacetimes," Class. Quant. Grav. 21, 289 (2004) [arXiv:gr-qc/0103106];

P. K. Townsend and M. Zamaklar, "The first law of black brane mechanics," Class. Quant. Grav. 18, 5269 (2001) [arXiv:hep-th/0107228].

[31] D. Kastor and J. Traschen, "Stresses and strains in the first law for Kaluza-Klein black holes," JHEP 0609, 022 (2006) [arXiv:hep-th/0607051]. 
[32] D. Kastor, S. Ray and J. Traschen, "The First Law for Boosted Kaluza-Klein Black Holes," JHEP 0706, 026 (2007) [arXiv:0704.0729 [hep-th]].

[33] T. Harmark and N. A. Obers, "Phase structure of black holes and strings on cylinders," Nucl. Phys. B 684, 183 (2004) [arXiv:hep-th/0309230].

[34] T. Harmark and N. A. Obers, "General definition of gravitational tension," JHEP 0405, 043 (2004) [arXiv:hep-th/0403103].

[35] L. D. Landau and E. M. Lifshitz, "Theory of elasticity," Pergamon Press (1970). 